\title{
Ladder-Biquad Filter Partitioning for On-chip Tuning
}

\author{
Drazen Jurisic \\ Faculty of Electrical Engineering and Computing \\ University of Zagreb \\ Unska 3, HR-10 000 Zagreb, Croatia \\ drazen.jurisic@fer.hr
}

\author{
George S. Moschytz \\ School of Engineering \\ Bar-Ilan University \\ IL-52 900 Ramat-Gan, Israel \\ moschytz@isi.ee.ethz.ch
}

\begin{abstract}
One of the problems with on-chip active-RC filters is that in order to satisfy tight specifications accurately, they need to be fine-tuned. This becomes more difficult with increasing filter order. In this paper, we introduce a simplified tuning method which is obtained by a new structure consisting of a filter section of second- or third-order (Biquad or Bitriplet which is referred to as a 'tuning block'), in cascade with a ladder-RLC filter (passive LC, or simulated-active RC). The cut-off frequency of the resulting filter can be simply tuned by adjusting one component of the tuning block. The new ladder tuning-block (LTB) is compared with two conventional structures, a cascade of Biquads and a single-ladder filter. Fine tuning these two structures is considerably more complicated. It is shown that beside the advantage of ease of tuning, the sensitivity to component tolerances of the new structure is lower than that of the two conventional ones, for the price of a slightly higher filter order.
\end{abstract}

Keywords-active-RC filters; analog filters; filter synthesis; filter tuning; elliptic filters; sensitivity

\section{INTRODUCTION}

It is well-known that LCR ladder filters that are equally terminated at both ends, have minimum sensitivity to passive component variations in the pass band (see [1]). This is true also if the LCR ladder filters are simulated by inductorless active-RC structures. (However, in this case, because there will be more components that can vary, the overall tolerance to component variations will be larger.) If the latter are manufactured on-chip, filter tuning will be imperative due to the wide tolerances of on chip components. In many cases, it is sufficient to adjust only the cut-off frequency of a filter, and if so, it is desirable to do so with only one trimming component. In this paper, we show that by replacing a conventional ladder filter, or cascade of Biquads, by a ladder filter of slightly lower order (e.g. $n-1$ or $n-2)$, cascaded with a 'tuning Biquad,' tuning of the cut-off frequency using just one trimming component becomes relatively simple [2], [3]. The trimming component is a resistor in the tuning Biquad. Because it is generally (but not always) preferable to realize the latter by a third-order filter section, we prefer to call this a 'tuning block' (TB).

\section{Filter PARTITIONING}

\section{A. Ladder Filters}

Before we discuss our 'ladder tuning-block' [LTB] filter partitioning method, it is useful to recall the basics of LC ladder filter design. We do so by reviewing a simple design example, and referring to the classical filter tables of A. I. Zverev and R. Saal [4], [5].

Consider, the filter specifications in Fig. 1 of a seventh-order elliptic or Chebyshev-Cauer low-pass filter with a reflection coefficient $\rho=25 \%$ (corresponding to $A_{\max }=0.280287$ ), a normalized stop-band edge frequency $\omega_{s}=1.30541$ (corresponding to $\Theta=50^{\circ}$ ) and a minimum stop band attenuation $A_{\min }^{0}=58 \mathrm{~dB}$. From the tables we obtain a filter defined by the designation CC 072550 . This filter has $A_{\min }=64.3615 \mathrm{~dB}$ which is larger than $A^{0}{ }_{\text {min }}$ (which was specified) and results in a 'safety-margin' of $\Delta A_{\min }=A_{\min }-A^{0}{ }_{\min }=6.3615 \mathrm{~dB}$. The resulting normalized voltage transfer function is given by

$$
\begin{aligned}
& T(s)=\frac{V_{\text {out }}}{V_{\text {in }}}=\frac{0.0044883 \cdot\left(s^{2}+1.76\right)}{(s+0.3764)\left(s^{2}+0.5822 s+0.3953\right)} \times \\
& \times \frac{\left(s^{2}+2.397\right)\left(s^{2}+6.50\right)}{\left(s^{2}+0.2918 s+0.8051\right)\left(s^{2}+0.08155 s+1.027\right)} .
\end{aligned}
$$

The corresponding filter topology with component values is given in Fig. 2, the amplitude characteristic in Fig. 3, and the simulated-active RC version in Fig. 4 below. Fig. 3 is simulated using the OrCAD PSpice 16 program [6] in which all frequencies are given in $\mathrm{Hz}$, e.g. the normalized cut-off frequency $\omega_{c}=1$ in Fig. 1, when divided by $2 \pi$ is $f_{c} \approx 0.16$.

The normalized transfer-function zeros, poles, and LCR component values are given in the filter tables. The zeros are the resonant frequencies of the parallel tanks: $\Omega_{2}=2.5494, \Omega_{4}=1.3266, \Omega_{6}=1.5482$. The pole $Q$ values are: $q_{p 1}=1.0799, q_{p 2}=3.0753$ and $q_{p 3}=12.43$.

\section{B. Partitioned Filters}

Filter partitioning, in the context of this paper, consists of finding two transfer functions $T_{L}(s)$ and $T_{B}(s)$ such that their product replaces an initial $n$ th-order transfer function $T(s)$, where the index ' $\mathrm{L}$ ' designates the ladder part, index ' $\mathrm{B}$ ' the tuning block, and ' $\mathrm{P}$ ' denotes the overall partitioned filter. Thus,

$$
T(s) \rightarrow T_{P}(s)=T_{L}(s) \cdot T_{B}(s) .
$$

The design starts with $A_{\max }, A^{0}{ }_{\min }, \omega_{s}$, and order $n$ of the original single-ladder filter (as discussed under $A$ above, and shown in Fig. 1). In distributing the pass-band ripple between the ladder filter and the

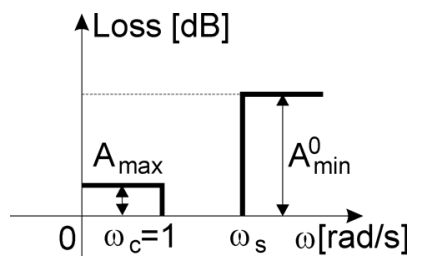

Figure 1. Example of filter specifications.

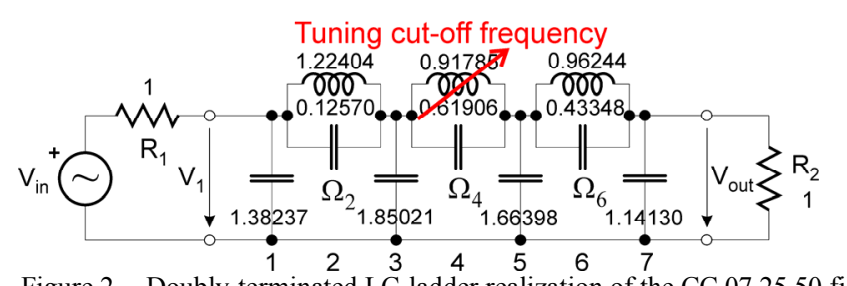

Figure 2. Doubly-terminated LC-ladder realization of the CC 072550 filter.

This work was supported by the Ministry of Science, Education and Sports of Republic of Croatia. 


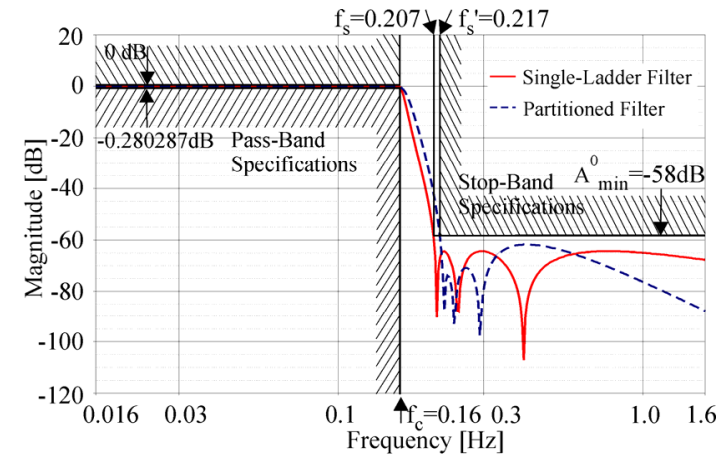

Figure 3. Amplitude characteristics that satisfy specifications in Fig.1.

tuning block, it can be shown that the optimum solution is to assign half to the tuning block and half to the ladder part, i.e. $A_{\max L}=A_{\max B}=$ $A_{\max } / 2$ [7].

i) The tuning-block design parameters. In general, it is recommended to use a third-order tuning block $\left(n_{B}=3\right)$, because it results in a minimum order for the ladder part of the partitioned filter. With $A_{\max B}=A_{\max } / 2=0.140144 \mathrm{~dB}$ and the filter tables (e.g. [4][5]), and with $\omega_{s}$, and $n_{B}$, we can calculate the tuning-block transfer function. Note that we use the same $\omega_{s}$ as in the ladder part [7]. Note also that with conventional filter tables, we are limited to integer values of $\rho$ with the corresponding $A_{\max }$. To obtain an "accurate" tuning block, a filter design program has been developed [8] that calculates the tuning block parameters with the exact $\rho$ value derived directly from the given $A_{\max B}$. This is more efficient in terms of filter complexity. Thus, referring to (3), with $A_{\max B}=0.140144 \mathrm{~dB}$, which corresponds to $\rho=17.82 \%$, and $\omega_{s}^{\prime}=1.3644$ (see footnote 1 below), we obtain $\omega_{p}=$
1.19257, $q_{p}=2.4984, \omega_{z}=1.50992, \gamma=1.26889, k_{B}=0.79155$ and $A_{\min B}=$ $12.911 \mathrm{~dB}$. The voltage transfer function of the tuning block results as:

$$
T_{B}(s)=\frac{k_{B} \cdot\left(s^{2}+\omega_{z}^{2}\right)}{(s+\gamma)\left(s^{2}+\frac{\omega_{p}}{q_{p}} s+\omega_{p}^{2}\right)}=\frac{0.79155 \cdot\left(s^{2}+2.28\right)}{(s+1.269)\left(s^{2}+0.4773 s+1.422\right)}
$$

The TB with component values is shown on the right of Fig. 5.

ii) The ladder-part design parameters. Starting with $A_{\max L}=A_{\max } / 2=0.140144 \mathrm{~dB}, \omega_{s}=1.30541$ (same $\omega_{s}$ as in the singleladder filter in $A$ ), and with $n_{B}=3$, we obtain the (rounded up) filter order $n_{L}=6$ from a nomograph that has been specially developed for the purpose of filter partitioning (see [7]).

To obtain the ladder-part transfer function and component values, we again use design programs that were specially developed for filter partitioning [8] and obtain $A_{\min L}=48.6858 \mathrm{~dB}$. The final partitioned filter has a minimum stop-band attenuation of $A_{\min P}=A_{\min L}+A_{\min B}=$ $61.5967 \mathrm{~dB}$ which satisfies the originally required $A_{\min }^{0}=58 \mathrm{~dB}$ very well (see Fig. 3). The voltage transfer function of the partitionedladder filter results as:

$$
\begin{gathered}
T_{L}(s)=\frac{0.05481661 \cdot\left(s^{2}+3.36847\right)}{\left(s^{2}+1.11891 s+0.423892\right)} \times \\
\times \frac{\left(s^{2}+1.96056\right)}{\left(s^{2}+0.558473 s+0.78977\right)\left(s^{2}+0.147864 s+1.08135\right)},
\end{gathered}
$$

where the pole $Q$ values are $q_{p 1}=0.58188, q_{p 2}=1.5913$ and $q_{p 3}=7.0327$. Note that these pole-Q values are lower than those of the original single-ladder filter. The partitioned ladder filter part with component values is shown on the left of Fig. 5, and the amplitude

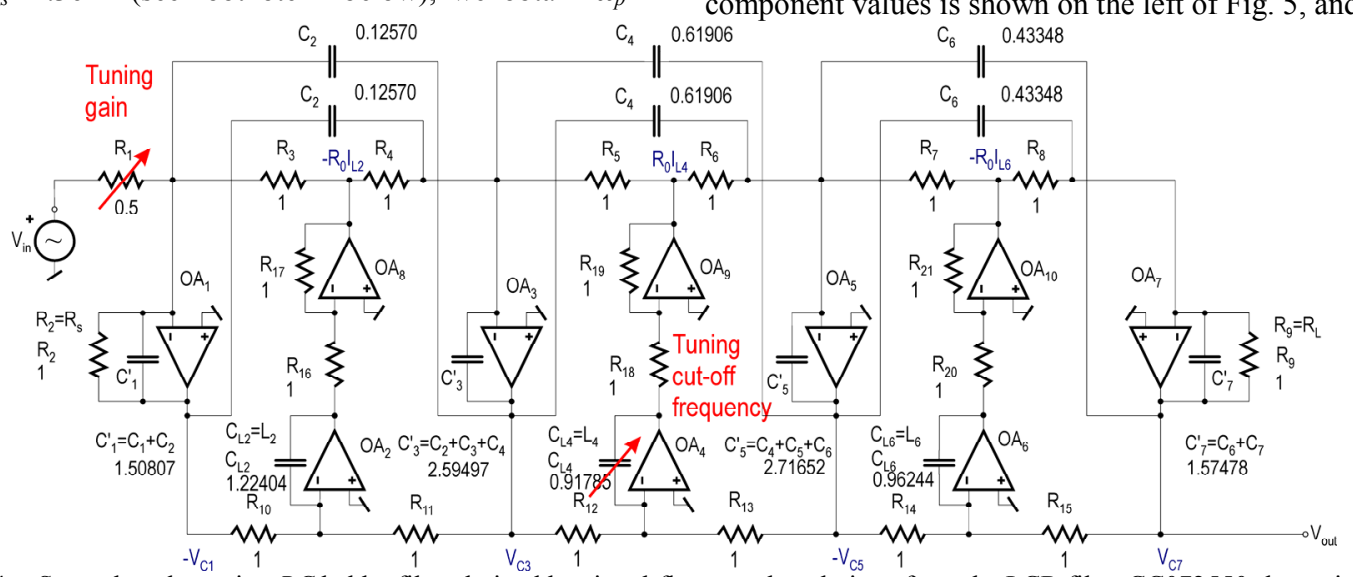

Figure 4. Seventh-order active- $R C$ ladder filter derived by signal-flow-graph technique from the LCR filter CC072550 shown in Fig. 2.

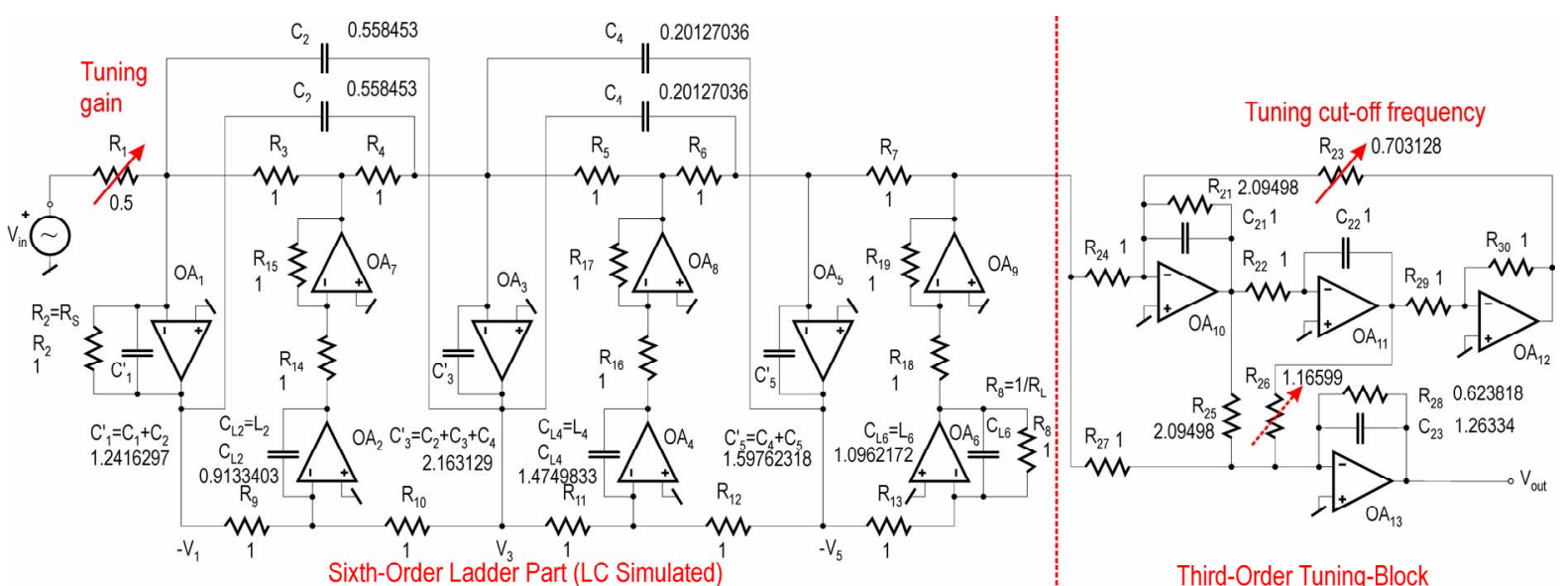

Figure 5. Active- $R C$ partitioned filter that satisfies the same specifications as the single-ladder filter CC 072550 . 


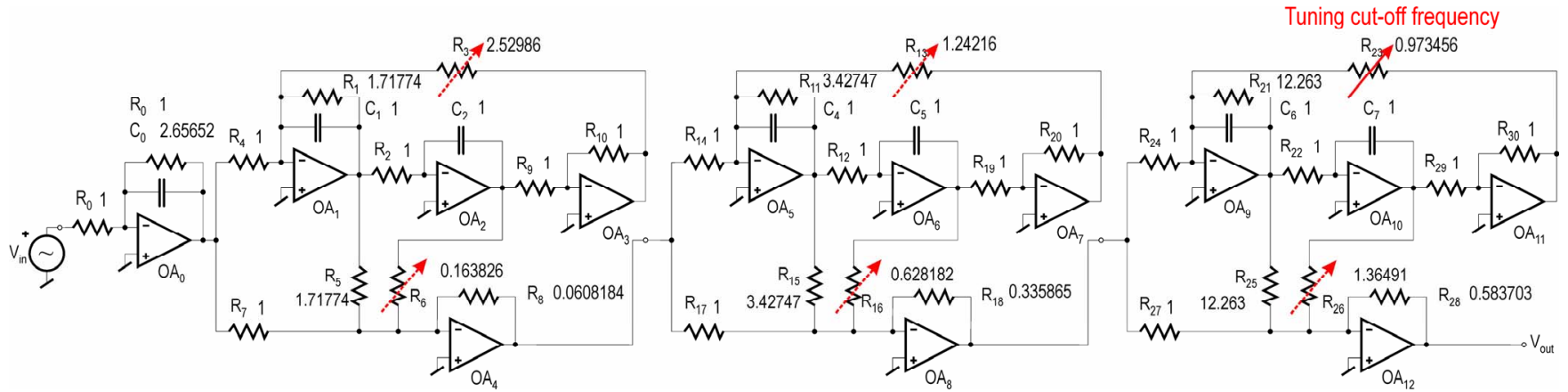

Figure 6. CC 072550 active-RC filter realized by a cascade of biquads.
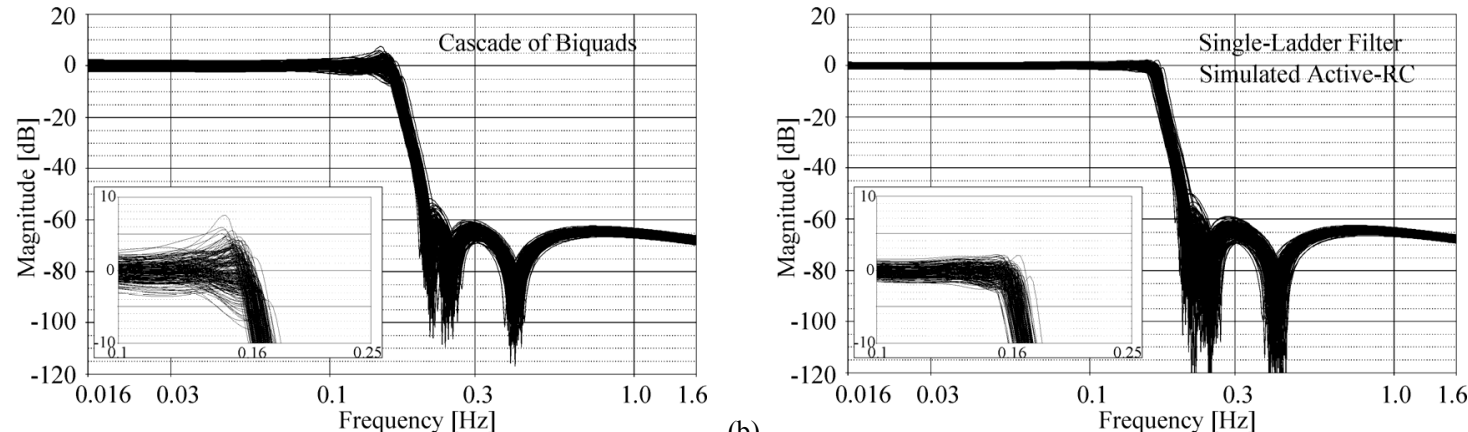

(a)

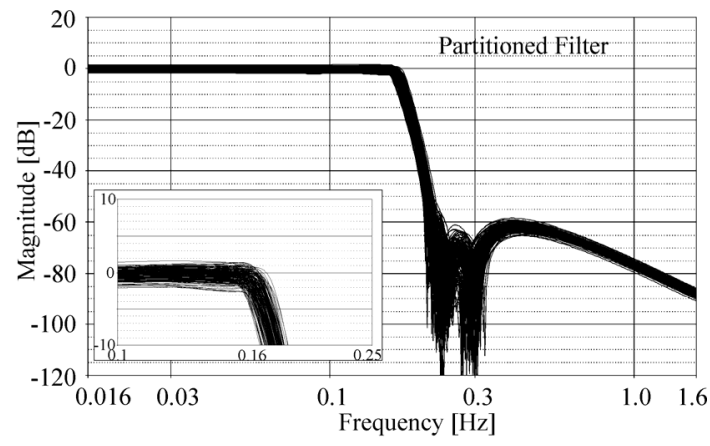

(b)

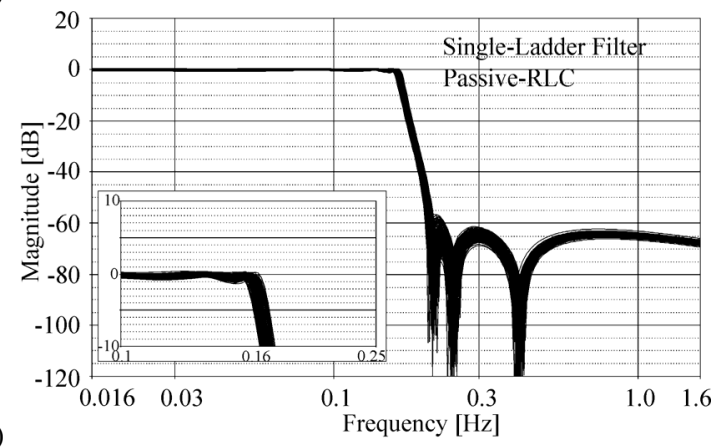

Figure 7. Monte Carlo runs of various realizations. (a) Cascade of Biquads. (b) Active-RC simulation of single-ladder RLC filter. (c) Partitioned filter. (d) Passive ladder-RLC filter. All simulations by PSpice.

response of the complete partitioned filter is compared with that of the original ladder filter in Fig. 3. ${ }^{1}$

Note that the order of the complete partitioned filter has increased to 9, whereas the order of the single-ladder filter is 7 . However, in terms of component count, the difference between the two is small.

\section{Sensitivity And Tuning Procedure for The Partitioned FILTER}

In the example above we have replaced the single-ladder CC 0725 50 filter by a partitioned filter i.e., a cascade of a sixth-order ladder filter and a third-order tuning block. To complete the comparison of filter types, we have also included the cascade of three Biquads (one of which must, of course, be third-order) realizing the same CC 0725 50 filter. It is shown in Fig. 6.

${ }^{1}$ Note that in the design of even-order elliptic filters using [8] we are able to design the ladder-LC network with equal resistive terminations at both ends. This is referred to as case ' $\mathrm{C}$ ' in [5]. This transformation increases the stop-band edge frequency of the ladder part from $\omega_{s}=1.30541$ to $\omega_{s}^{\prime}=1.3644$. This slight increase in $\omega_{s}$ will generally be negligible due to the inherent design margin. If this is not the case, it must be compensated for by choosing a smaller initial $\omega_{s}$.
Comparing the sensitivities of all three filters with Monte Carlo runs using PSpice, while assuming a zero-mean uniform distribution and 5\% standard deviation for all components, we obtain the ensemble of responses shown in Fig. 7. The spread of the ensemble of responses for each filter is an indication of its sensitivity to component tolerances. However, it is also an indication of the number of components in a given circuit; the fewer the number of components in a circuit, the smaller the spread of responses resulting from component tolerances.

The spread of responses in the pass-band is highest for the Biquad cascade (Fig. 7a), due to the combination of relatively high sensitivity and component count. The active-RC simulated version of the original LC ladder network (Fig. 7b) is next. Here the large spread is a result of the large component count, coupled with the fact that the pole Qs are relatively high (the highest being 12.43); it is known that network sensitivity increases with pole Q [2]. In terms of curve-spread in the pass-band, the partitioned filter comes next (Fig. 7c). Here, it is the smaller pole Qs (the highest is 7.03269) with the resulting decrease in component-variation sensitivity that is responsible for the smaller spread of curves in the pass-band. Note that the price for this decrease is a relatively small increase in filter order (from 7 to 9), which is achieved with a small number of additional components. Finally, the smallest spread of pass-band curves is obtained with the doublyterminated passive LC ladder filter which, according to established 

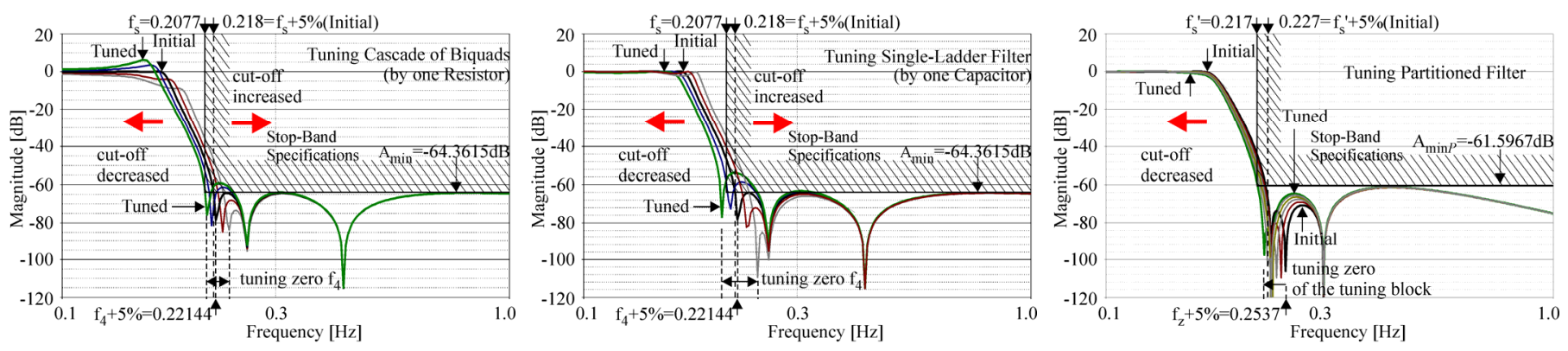

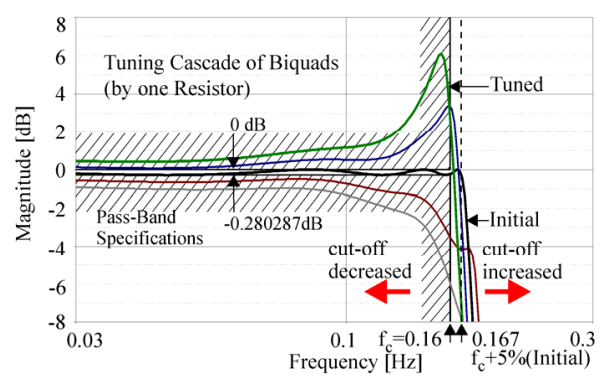

(a)

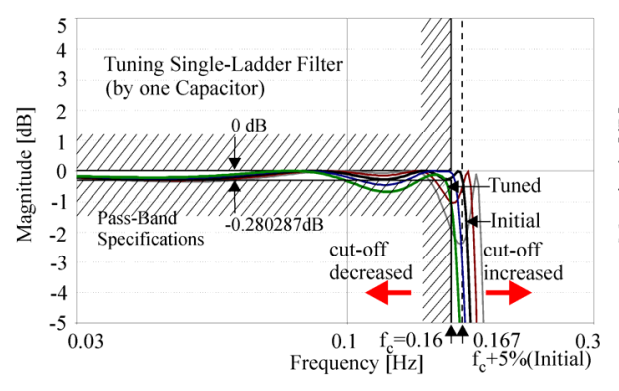

(b)

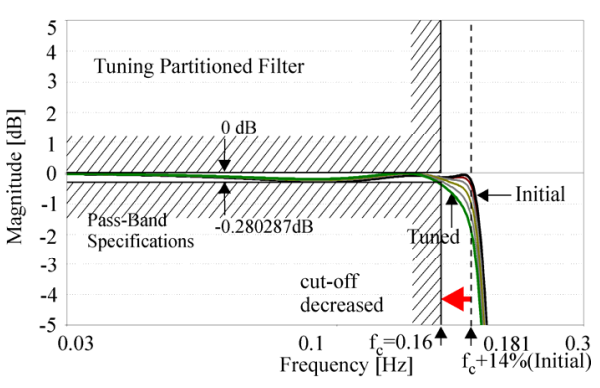

(c)

Figure 8. Tuning of cut-off frequency by $10 \%$. (a) Cascade of Biquads when one resistor $R_{3}$ (in max $\mathrm{Q}$ section) is tuned. (b) Active-RC simulation of singleladder RLC filter when capacitor representing $L_{4}$ is tuned. (d) Partitioned filter when $R_{3}$ and $R_{6}$ in the tuning block are tuned.

theory (see Orchard's theorem in [1]), has the smallest sensitivity of all (Fig. 7d).

The MC runs in Fig. 7 show that component tolerances in any of the filters will, among other things, shift the cut-off frequency horizontally in either direction by a significant amount (in our case $\pm 5 \%)$. Very often, the correct location of the cut-off frequency is the determining factor for the accuracy of a filter. Thus, tuning for the correct cut-off frequency, while maintaining adherence to the specifications, e.g. $A_{\max }, A_{\min }$ etc. of the initial filter, is sufficient to establish the correct performance of a filter. It is this assumption that has led us to concentrate our tuning effort on achieving the correct cut-off frequency with as few trimming components as possible. As we now show, only the partitioned filter with its "frozen" partial ladder filter and tuning block permits us to tune the cut-off frequency accurately. It does this with only one, or at most two, tuning components, while maintaining all other specifications.

Starting with the Biquad cascade, we performed a parametric analysis using the OrCAD PSpice 16 program by changing the value of $R_{3}$ of the max-Q Biquad in several steps, in an interval of $\pm 18 \%$ of the nominal value. This results in a cut-off frequency shift of $\pm 5 \%$ as shown in Fig. 8(a). However, it is clear that the specifications in the pass- and stop-band are unacceptably violated. To stay within specs, six resistors would have to be trimmed, i.e. two in each Biquad. Similarly, tuning the cut-off frequency of the LC ladder filter or its active-RC simulation by the single component that determines the lowest zero $\left(L_{4}\right.$ in the passive-RLC network, or the corresponding capacitor in the active realization) by $\pm 18 \%$, we obtain the tuning curves in Fig. 8(b). Here again, the cut-off frequency is shifted by $\pm 5 \%$, but the specifications are violated both in the pass-band and stop-band.

Finally, tuning the partitioned filter with only resistor $R_{3}$ of the tuning block, leads to a satisfactory solution (see Fig. 8c). Increasing the resistor $R_{3}$ of the tuning block by $25 \%$ (when the initial value of $R_{3}$ is deliberately low, e.g., by $40 \%$ ) and thus shifting the whole characteristic to lower frequencies, e.g. by $10 \%$, we can decrease the cut-off frequency by a required amount, while maintaining all filter specifications. In this case, there is a change in gain which, if necessary, can be adjusted for with $R_{6}$ of the tuning block. Other possibilities for gain adjustment exist, e.g., $R_{1}$ of the ladder filter part (see left side in Fig. 5).

\section{CONCLUSIONS}

A procedure for the design of low-sensitivity and tunable active- $\mathrm{RC}$ LP filters with finite (or infinite) zeros is presented. It consists of partitioning the transfer function of a given $n$ th-order, doublyterminated, LCR ladder filter into the cascade of two parts: the first is a doubly-terminated RLC ladder filter (which is realized by an active$\mathrm{RC}$ ladder simulation) and the second is a tuning block (Biquad or Bitriplet). Depending on the filter specifications, the overall order of the partitioned filter will be $n+1, n+2$, or $n+3$. The partitioned filter demonstrates simplified tuneability and decreased sensitivity properties for the price of a slightly higher filter order and complexity. Clearly, similar partitioning into ladder tuning-block cascades can be carried out for other filter types, e.g. highpass, bandpass, or bandstop.

\section{REFERENCES}

[1] H. J. Orchard, "Inductorless filters," Electron. Letters, vol. 2, no.6, pp. 224-225, June 1966.

[2] G. S. Moschytz and P. Horn, Active Filter Design Handbook. Chichester, U.K.: John Wiley and Sons, 1981.

[3] D. Jurisic, N. Mijat and G. S. Moschytz, "Tuning Elliptic Filters with a 'Tuning Biquad'," in Proceedings of ISCAS 2009, Taipei, Taiwan, May 24-27, 2009, pp. 45-48.

[4] A. I. Zverev, Handbook of Filter Synthesis. New York: John Wiley and Sons, 1967.

[5] R. Saal, Handbook of Filter Design. Berlin: AEG-Telefunken, 1979.

[6] PSpice Circuit Analysis - Users Manual, Microsim Corporation, Irvine CA-USA, 1996.

[7] D. Jurisic, G. S. Moschytz, "Ladder, Tuning-Block Partitioned Filters for On-chip Design," International Journal of Circuit Theory and Applications (submitted for publication).

[8] D. Jurisic, Filterpart and Filtersyn-Elliptic filter design programs for Matlab. Zagreb, Croatia: Faculty of El. Eng. and Computing, University of Zagreb, 2010. Available: http://maja.zesoi.fer.hr/ jurisic/Projects/ 